\title{
ASSOCIAÇÃO ENTRE LESÕES DA MUCOSA GASTRODUODENAL E HELICOBACTER PYLORI EM PACIENTES COM DOENÇA HEPÁTICA CRÔNICA
}

\author{
Rozangela Maria de Almeida Fernandes WYSZOMIRSKA, Laércio Tenório RIBEIRO, \\ Jackellyne Santos MONTEIRO, Bruno Pontes de Miranda VIDAL, \\ Roberta Maria Pereira Albuquerque de MELO e Cláudio Torres MIRANDA
}

\begin{abstract}
RESUMO - Contexto - A relação entre a infecção por Helicobacter pylori e lesões da mucosa gastroduodenal em pacientes com doença hepática crônica permanece controversa. Objetivo - Avaliar a presença de lesões da mucosa gastroduodenal e sua relação com Helicobacter pylori em pacientes com doença hepática crônica. Métodos - Estudaram-se 46 pacientes e 27 controles com dispepsia funcional, submetidos a endoscopia digestiva alta. Foram consideradas lesões da mucosa gastroduodenal a gastropatia da hipertensão portal, erosão e úlcera péptica. O Helicobacter pylori foi detectado através de duas amostras de biopsia do antro e do corpo gástrico, pelo método de Giemsa. Resultados - As lesões da mucosa gastroduodenal foram identificadas em 38 (82,6\%) pacientes com doença hepática crônica, significantemente mais frequente que nos controles $(P=0,02)$. A presença de Helicobacter pylori foi observada em 13 $(28,2 \%)$ dos pacientes com doença hepática e em $17(62,9 \%)$ dos controles. A estimativa de risco mostrou interação significante entre lesão da mucosa e doença hepática crônica ( $P=0,04$; OR 5,1 IC 95\%, 1,6-17,3). Quando associada à presença do Helicobacter pylori, o risco foi mais elevado na ausência da bactéria ( $P=0,005$; OR 13,0 IC 95\%, 1,4-327,9). Conclusão - Pacientes com doença hepática crônica mostram risco aumentado de desenvolver lesões da mucosa gastroduodenal, independente da presença de Helicobacter pylori. DESCRITORES - Infecções por helicobacter. Mucosa gástrica. Hepatopatias.
\end{abstract}

\section{INTRODUÇÃO}

As doenças hepáticas crônicas (DHC) apresentam elevada incidência e prevalência em todo o mundo, sendo observadas alterações da mucosa gastroduodenal, com risco elevado para o aparecimento de úlcera duodenal em cirróticos $^{(8,24,26,31)}$. Dentre os mecanismos fisiopatológicos postulados, destacam-se as alterações séricas da gastrina ${ }^{(13,29)}$, com consequente aumento da secreção ácida, mudanças no fluxo sanguíneo ${ }^{(28)} \mathrm{e}$ diminuição da secreção de prostaglandina na mucosa gástrica $^{(1)}$, sendo ainda considerada a presença do Helicobacter pylori $(\text { H. pylori })^{(10,18)}$.

A infecção pelo $H$. pylori é altamente prevalente, principalmente em países em desenvolvimento ${ }^{(15)}$, sendo responsável por lesões como erosões e úlceras gastroduodenais. Em pacientes com DHC sua prevalência é controversa ${ }^{(7,9,22,32,35,36)}$, bem como a existência de associações com lesões da mucosa gastroduodenal $(\mathrm{LMGD})^{(5)}$.

A gastropatia da hipertensão portal $(\mathrm{GHP})^{(3,30)}$ está associada primordialmente à hipertensão portal, caracterizando-se endoscopicamente por áreas com aspecto em mosaico e pontos vermelhos no centro, predominantes no corpo e fundo gástricos ${ }^{(21)}$. Além disso, pode estar associada a erosões e úlceras, que apresentam características endoscópicas semelhantes àquelas encontradas em pacientes sem hipertensão portal.

O conhecimento da prevalência da infecção pelo $H$. pylori em pacientes com DHC e o estudo da associação, ou não, com as lesões gastroduodenais, podem ser úteis na melhor caracterização da patogênese da GHP e na avaliação de possível efeito aditivo na produção de lesões gastroduodenais, quando estão presentes as duas condições. VERGARA et al. ${ }^{(34)}$, em estudo de metanálise, incluindo sete estudos que avaliaram a prevalência da infecção pelo $H$. pylori e lesões endoscópicas associadas em cirróticos, concluíram que a infecção pelo microorganismo esteve presente em $60,7 \%$ dos indivíduos avaliados, com aumento do risco do aparecimento de úlcera péptica. Entretanto, BATMANABANE et al. ${ }^{(2)}$ concluíram que a GHP não proporciona ambiente favorável à colonização

Departamento de Clínica Médica/Hospital Universitário Professor Alberto Antunes, Universidade Federal de Alagoas, Maceió, AL.

Correspondência: Dra. Rozangela Maria de Almeida Fernandes Wyszomirska - Rua Desportista Humberto Guimarães, $342 / 201$ - Ponta Verde - 57035 - 030 - Maceió, AL, Brazil. E-mail: rozangelaw@yahoo.com.br 
pelo $H$. pylori, sugerindo não haver contribuição da bactéria na patogênese da GHP.

O objetivo deste estudo foi avaliar a presença de lesões da mucosa gastroduodenal e sua relação com $H$. pylori em pacientes com DHC.

\section{MÉTODO}

\section{Pacientes}

Realizou-se estudo transversal em que foram incluídos pacientes com DHC por diversas causas, com indicação para realização de endoscopia digestiva alta por suspeita de hipertensão portal e indivíduos com dispepsia funcional, como grupo controle, no Ambulatório de Hepatologia do Hospital Universitário Professor Alberto Antunes da Universidade Federal de Alagoas, Maceió, AL, no período de janeiro de 2005 a janeiro de 2006.

Os critérios de exclusão foram: pacientes com uso prévio (até 1 mês) ou atual de antibióticos, terapia prévia de erradicação do H. pylori, carcinoma hepatocelular ou outras neoplasias e episódio recente (máximo de 2 semanas) de hemorragia digestiva alta. O estudo foi aprovado pelo Comitê de Ética em Pesquisa da Universidade Federal de Alagoas e foi executado de acordo com a Declaração de Helsinki.

\section{Diagnóstico da doença hepática crônica}

As doenças hepáticas foram identificadas como cirrose hepática secundária aos vírus das hepatites $\mathrm{B}$ e C, esquistossomose mansônica e doença mista, quando havia associação da etiologia viral com Schistosoma mansoni. $\mathrm{O}$ diagnóstico da cirrose hepática foi realizado através de exame físico, alterações de exames laboratoriais, achados de ultrassonografia e/ou biopsia hepática. A infecção pelo vírus $\mathrm{C}$ foi detectada pela presença de anticorpos anti-HCV de segunda geração e confirmada pelo HCV-RNA, enquanto que para a hepatite B foi considerada a presença do HBSAg. O Schistosoma mansoni foi identificado pela presença de ovos em amostra de fezes, através do método de Kato Katz, ou pesquisa de ovos na mucosa retal e a doença hepática esquistossomótica, forma hepatoesplênica, foi confirmada por achados de exame físico e de ultrassonografia.

\section{Diagnóstico endoscópico de lesões da mucosa gastroduodenal}

Para a realização de endoscopia digestiva alta os pacientes e controles foram submetidos a sedação com meperidina e midazolam, em doses que variaram de acordo com a tolerância, visando atingir nível de sedação consciente. O exame foi realizado pelo mesmo examinador, utilizando aparelho de videoendoscopia Olympus. Foram admitidas como LMGD a erosão, úlcera e gastropatia da hipertensão portal. O diagnóstico endoscópico de erosão caracterizou-se pela identificação de solução de continuidade tecidual, plana ou elevada. A úlcera foi identificada como lesão superficial da mucosa com base recoberta por fibrina e bordas distintas e a GHP através da presença de áreas da mucosa com aspecto em mosaico e pontos vermelhos no centro, predominantes no corpo e fundo gástricos ${ }^{(16)}$. Em todos os pacientes foram realizadas biopsias, sendo retirados dois fragmentos do antro e do corpo. O material colhido foi imediatamente fixado em formalina a $10 \%$.

\section{Diagnóstico histopatológico}

Os fragmentos colhidos durante o exame endoscópico foram incluídos em parafina e corados pelos métodos da hematoxilina-eosina e Giemsa, sendo o primeiro para avaliação de infiltrado inflamatório e o segundo para pesquisa do H. pylori. Todas as lâminas foram estudadas pelo mesmo patologista.

\section{Análise estatística}

Os dados foram construídos e analisados no banco de dados Epi-Info Windows. As medidas de associação foram: estimativa do risco relativo - odds ratio (OR) e qui ao quadrado corrigido. O OR foi utilizado para pesquisar a intensidade das associações. O intervalo de confiança considerado foi de $95 \%$.

\section{RESULTADOS}

Foram incluídos no estudo 73 indivíduos, sendo 46 pacientes com DHC (39 do gênero masculino e 7 do feminino, média de idade de 45,6 $\pm 12,8$ anos), e 27 indivíduos do grupo controle, sem doença hepática mas com dispepsia (19 do gênero masculino e 8 do feminino, média de idade de 45,2 $\pm 14,4)$.

As doenças hepáticas crônicas identificadas foram: cirrose hepática secundária à hepatite B e C em 30 (65,22\%), esquistossomose mansônica em $7(15,22 \%)$ e doença mista em $9(19,56 \%)$ pacientes.

Trinta e oito $(82,6 \%)$ dos 46 pacientes com DHC e 13 $(48,1 \%)$ dos 27 controles apresentaram LMGD, sendo observada diferença estatisticamente significante $(P<0,05)$. Os tipos de LMGD observados nos pacientes e controles encontram-se na Tabela 1.

TABELA 1. Lesões de mucosa gastroduodenal em pacientes com doença hepática crônica e controles

\begin{tabular}{|c|c|c|c|c|c|}
\hline Lesões de mucosa & DHC $(n=46)$ & $\%$ & Controles $(\mathrm{n}=27)$ & $\%$ & $P$ \\
\hline Presença de LMGD & 38 & 82,6 & 13 & 48,1 & $<0,05^{*}<$ \\
\hline Ausência de LMGD & 08 & 17,4 & 14 & 51,9 & \\
\hline GHP & $16 / 38$ & 42,1 & $3 / 13$ & 23,05 & $<0,05 *$ \\
\hline Erosão de antro e/ou duodeno & $11 / 38$ & 28,9 & $10 / 13$ & 76,95 & NS \\
\hline Úlcera péptica & $04 / 38$ & 10,5 & $00 / 13$ & - & \\
\hline Associação GHP e LMGD & $07 / 38$ & 18,4 & $00 / 13$ & - & \\
\hline
\end{tabular}


Ao se avaliar a estimativa do risco de ocorrer lesões da mucosa gastroduodenal em pacientes com DHC, foi observado um coeficiente elevado (OR 5,12 IC 95\%, 1,55-17,37), sendo essa associação estatisticamente significante $(P=0,002)$.

Dos 46 pacientes com DHC, $13(28,2 \%)$ foram $H$. pylori positivo e dos 27 indivíduos do grupo controle, 17 (62,9\%) foram positivos, sendo observada diferença estatisticamente significante $(P=0,008)$.

As características demográficas e tipos de lesões dos pacientes com DHC e dos controles, associados à presença do H. pylori estão demonstradas na Tabela 2. Em relação ao grupo de doentes com DHC não foi observada diferença significante entre $H$. pylori positivo e negativo, em relação à frequência, idade, gênero e nem quanto ao tipo de lesões gastroduodenais, no entanto com grupo controle a presença de H. pylori foi fator significante para o aparecimento de erosões.

TABELA 2. Características de pacientes com doença hepática crônica e controles em relação à presença de $H$. pylori

\begin{tabular}{lccc}
\hline Características & H. pylori positivo & H.pylori negativo & $\boldsymbol{P}$ \\
\hline DHC & $\mathrm{n}=13$ & $\mathrm{n}=33$ & \\
Idade (anos) & $45,85 \pm 18,53$ & $44,06 \pm 17,67$ & $\mathrm{NS}$ \\
Gênero (M/F) & $11 / 02$ & $28 / 05$ & $\mathrm{NS}$ \\
Ausência LMGD & 03 & 13 & $\mathrm{NS}$ \\
Presença LMGD & 10 & 20 & $\mathrm{NS}$ \\
GHP isolada & 04 & 08 & $\mathrm{NS}$ \\
Erosões gastroduodenais & 03 & 06 & $\mathrm{NS}$ \\
Úlcera péptica & 01 & 03 & $\mathrm{NS}$ \\
Associação GHP e LMGD & 02 & 03 & $\mathrm{NS}$ \\
Controles & $\mathrm{n}=17$ & $\mathrm{n}=10$ & \\
Idade (anos) & $44,22 \pm 16,55$ & $45,02 \pm 17,58$ & $\mathrm{NS}$ \\
Gênero (M/F) & $12 / 05$ & $07 / 03$ & $\mathrm{NS}$ \\
Ausência LMGD & 05 & 09 & $\mathrm{NS}$ \\
Presença LMGD & 12 & 01 & $P<0,05 *$ \\
GHP isolada & 03 & 00 & $\mathrm{NS}$ \\
Erosões gastroduodenais & 09 & 01 & NS \\
Úlcera péptica & 00 & 00 & NS \\
Associação GHP e LMGD & 00 & 00 & $\mathrm{NS}$ \\
\hline H. pylori = Helicobacter pylori; M $=$ masculino; F $=$ feminino; LMGD = lesão de mucosa gastroduodenal; GHP = gastropatia da
\end{tabular}

hipertensão portal; $\mathrm{NS}=$ = não-significante; $* P<0,05$ significante

A Tabela 3 mostra que a associação entre lesão na mucosa gástrica e a DHC tende a ocorrer apenas na ausência do $H$. Pylori $(P=0,005$; OR 13,0 IC 95\%, 1,4-327,9).

TABELA 3. Estimativa de risco relativo para presença de lesão na mucosa gastroduodenal em pacientes com doença hepática crônica ajustada pela presença de $H$. pylori $(\mathrm{n}=73)$

\begin{tabular}{lcccc}
\hline & & OR & IC95\% & $\boldsymbol{P}$ \\
\hline Sem ajuste & & 5,1 & $(1,6-17,3)$ & $0,002^{*}$ \\
Ajustado pela presença de H. pylori & Positivo & 1,4 & $(0,0-9,9)$ & 0,51 \\
& Negativo & 13,0 & $(1,4-327,9)$ & $0,005 *$ \\
\hline
\end{tabular}

H. pylori $=$ Helicobacter pylori; $* P<0,05$ significante

\section{DISCUSSÃO}

Embora o papel da infecção pelo $H$. pylori na úlcera péptica $^{(34)}$, câncer gástrico ${ }^{(39)}$, gastropatia congestiva ${ }^{(37)}$ e encefalopatia hepática ${ }^{(38)}$ tenham sido investigados em pacientes com cirrose hepática, os dados disponíveis ainda são escassos e contraditórios ${ }^{(40)}$.

A prevalência de úlcera péptica em pacientes com cirrose hepática vem sendo identificada entre $6 \%$ a $33 \%{ }^{14}$ 27), sendo também os pacientes com maior associação com a gastropatia da hipertensão portal ${ }^{(27)}$. Neste estudo, observou-se que $80,31 \%$ dos pacientes com DHC apresentaram LMGD à endoscopia digestiva alta, sendo que $16(42,1 \%)$ apresentavam GHP, $7(18,4 \%)$ associação de lesões e $5(10,5 \%)$ úlcera péptica.

A prevalência de $H$. pylori em pacientes com cirrose apresenta grande variação, entre $26 \%$ e $89 \%{ }^{(17,19)}$. Ao se avaliar os efeitos da erradicação de $H$. pylori em doentes com cirrose hepática, LO et al. ${ }^{(14)}$ observaram prevalência da bactéria de $52 \%$ e QUEIROZ et al. ${ }^{(25)}$ avaliando pacientes portadores de vírus C encontraram prevalência de $H$. pylori de 70,2\% em cirróticos e $47,5 \%$ em pacientes não-cirróticos. No presente estudo, encontrou-se prevalência de $H$. pylori de $28,2 \%$ em pacientes com DHC por diversas causas e de $62,7 \%$ no grupo controle, de indivíduos dispépticos. Em estudo de metanálise, GISBERT et. al..$^{(9)}$ observaram resultados semelhantes, com prevalência do $H$. pylori em pacientes com hepatopatia crônica e lesão de mucosa gastroduodenal, igual ou até menor, quando comparado aos pacientes com lesão de mucosa gastroduodenal e sem doença hepática crônica. Fator que poderia contribuir com os resultados discordantes entre os estudos seria a utilização de métodos diferentes para a identificação do H. pylori, inclusive a sorologia ${ }^{(22,23)}$, que em pacientes cirróticos pode superestimar a prevalência da bactéria, já tendo sido demonstrada baixa sensibilidade e especificidade nesse grupo de pacientes ${ }^{(40)}$.

Outro aspecto importante no presente estudo é que não foi observada diferença significante entre os grupos $H$. pylori negativo e $H$. pylori positivo, em relação à média de idade, gênero, presença ou ausência de lesões da mucosa gastroduodenal, independente do tipo de lesão. Esses resultados foram semelhantes aos encontrados por LO et al..$^{(14)}$. No entanto, CHEN et al. ${ }^{(4)}$ encontraram uma expressão do antígeno de $H$. pylori na mucosa de pacientes com DHC em $69 \%$ dos pacientes, sendo que a extensão e a intensidade de inflamação crônica do antro foram observadas em $95,5 \%$ dos pacientes com DHC, recomendando o tratamento precoce da bactéria nesse grupo específico de doentes.

Os resultados desta casuística demonstraram uma associação entre LMGD e DHC, com risco de 5,1 vezes para o aparecimento das lesões, mas a análise estratificada mostrou que essa associação é mais intensa quando o $H$. pylori esteve ausente. Esse resultado sugere que o $H$. pylori pode não ser fator de risco fundamental para o aparecimento de LMGD em pacientes com DHC, devendo, no entanto, ter papel secundário, que necessita ser definido. QUEIROZ et al.(25) demonstraram em seu estudo que a infecção por $H$. pylori e a idade foram fatores associados com cirrose hepática em portadores de vírus $\mathrm{C}$, sugerindo que pacientes com cirrose hepática secundária à infecção pelo vírus $\mathrm{C}$ podem ser mais 
susceptíveis à infecção pelo $H$. pylori, que em pacientes sem cirrose hepática. Os pacientes com DHC, independente da causa, podem ter alterações na resposta imune ${ }^{(6,11,20)}$, aumentando a susceptibilidade de adquirir infecções. Além disso, são pacientes submetidos a endoscopia digestiva alta com mais frequência que a população geral ${ }^{(26,29)}$, fator esse que aumenta o risco de adquirir infecção por $H$. pylori ${ }^{(12,33)}$. Por fim, a hipertensão portal que se instala em pacientes com DHC não pode ser negligenciada, já tendo sido demonstrado que pacientes com cirrose e história de sangramento digestivo têm mortalidade maior que os pacientes sem cirrose. CHEN et al. ${ }^{(4)}$ observaram que a incidência de úlcera péptica aumenta em $44 \%$, quando a cirrose hepática associa-se com a presença de varizes de esôfago.

\section{CONCLUSÕES}

Os resultados demonstram interação significante entre a associação de LMGD em pacientes com DHC, independente da causa desta última e da presença do H. pylori. Esses resultados estimulam a realização de futuros estudos para melhor clarear o papel do $H$. pylori na patogênese da doença hepática e o estabelecimento das possíveis relações com a hipertensão portal, resposta imune e outros.

Wyszomirska RMAF, Ribeiro LT, Monteiro JS, Vidal BPM, Melo RMPA, Miranda CT. Association between Helicobacter pylori infection and gastroduodenal lesions in patients with chronic liver disease. Arq Gastroenterol. 2009;46(4):256-60.

ABSTRACT - Context - The relationship between Helicobacter pylori infection and gastroduodenal lesions in chronic liver disease remains controversial. Objective - Evaluate the evidence of the role of H. pylori infection in gastroduodenal lesions in patients with chronic liver disease. Methods - Fortysix patients with chronic liver disease were matched with 27 dyspeptic persons for age and sex. The gastroduodenal lesions were portal hypertension gastropathy, erosion and peptic ulcer. All patients underwent upper endoscopy: two biopsies were taken in the antrum and in the gastric body. The biopsies were used for Giemsa staining. Results - A gastroduodenal lesions were found in $38(82.6 \%)$ patients with liver disease and was significantly more frequent than among controls $(P=0.002)$. H. pylori infection was detected at histological assessment in $13(28.2 \%)$ patients with chronic liver disease and in $17(62.9 \%)$ controls. The odds ratio (OR) showed an interaction statistically significant between gastroduodenal lesions and chronic liver disease $(P=0.04 ; \mathrm{OR}=5.1 ; 95 \% \mathrm{CI}=1.6-17.3)$. When adjusted for the presence of $H$. pylori OR was significantly with $H$. pylori negative $(\mathrm{OR}$ 13.0 IC 95\%, 1.4-327.9). Conclusion - Patients with chronic liver disease showed higher risk of developing gastroduodenal lesions regardless of the presence of the $H$. pylori infection.

HEADINGS - Helicobacter infections. Gastric mucosa. Liver diseases.

\section{REFERÊNCIAS}

1. Arakawa T, Satoh H, Fukuda T, Nakamura H, Kobayashi K. Endogenous prostaglandin E2 in gastric mucosa of patients with alcoholic cirrhosis and portal hypertension. Gastroenterology. 1987;93:135-40.

2. Batmanabane V, Kate V, Ananthakrishnan N. Prevalence of Helicobacter pylori in patients with portal hypertensive gastropathy - a study from South India. Med Sci Monit. 2004;10;133-6.

3. Chaves DM, Sakai P, Mucenic M, Ishioka, S. Comparative study of portal hipertensive gastropathy in schistosomiasis and hepatic cirrhosis. Endoscopy. 2002;34:199-202.

4. Chen JJ, Changchien CS, Tai DI, Chiou SS, Lee CM, Kuo CH. Role of Helicobacter pylori in patients with cirrhosis with peptic ulcer. A serological study. Dig Dis Sci. 1994;39:1565-8.

5. Cheung RC, Cooper S, Keeffe EB. Endoscopic gastrointestinal manifestation of liver disease. Gastrointest Endosc Clin N Am. 2001;11:15-44.

6. Crabtree JE, Shallcroos TM, Heatley RV, Wyatt JI. Mucosal tumor necrosis factor $\mathrm{x}$ interleucin-6 in patients with Helicobacter pylori associated gastritis. Gut. 1991;32:1473-7.

7. D’Amico G, Montalbano L, Traina M, Pisa R, Menozzi M, Spano C, Pagliaro L. Natural history of congestive gastropathy in cirrhosis. The Liver Study Group of V. Cervello Hospital. Gastroenterology. 1990;99:1558-64.

8. Dore MP, Mura D, Deledda S, Maragkoudakis E, Pironti A, Realdi G. Active peptic ulcer disease in patients with hepatitis $\mathrm{C}$ virus-related cirrhosis: the role of Helicobacter pylori infection and portal hypertensive gastropathy. Can J Gastroenterol. 2004;18:521-4

9. Gisbert JP, Moreno-Otero R, Pajares JM. Prevalência de infeccion por Helicobacter pylori em la hepatopatia crônica y relación com sus complicaciones: revision sistemática y metaanálisis. Med Clin (Barc). 2002;119:464-74.

10. Hopkins RJ, Girardi LS, Turney EA. Relationship between Helicobacter pylori eradication and reduced duodenal and gastric ulcer recurrence: a review. Gastroenterology. 1996;110:1244-52.
11. Karttunen R, Karttunen T, Ekre HP, MacDonald TT. Interferon gamma and interleukin-4 secreting cells in the gastric antrum in Helicobacter pylori positive and negative gastritis. Gut. 1995;36:341-5.

12. Langenberg W, Raws EAJ, Oudbier JH, Tytgat GN. Patient-to patient transmission of Campylobacter pylori infection by fiberoptic gastroduodenoscopy and biopsy. J Infect Dis. 1990;161:507-11.

13. Lenz HJ, Hogan DL, Isenberg JI. Intestinal phase of gastric acid secretion in humans with and without portocaval shunt. Gastroenterology. 1985;89:791-6.

14. Lo GH, Yu HC, Chan, YC, Chen WC, Hsu P, Lin CK, Lai KH. The effects of eradication of Helicobacter pylori on the recurrence of duodenal ulcers in patients with cirrhosis. Gastrointest Endosc. 2005;62:350-6.

15. Malaty HM. Epidemiology of Helicobacter pylori infection. Best Pract Res Clin Gastroenterol. 2007;21:205-14.

16. McCormack TT, Sims J, Eyre-Brook I, Kennedy H, Goepel J, Johnson AG, Triger DR. Gastric lesions in portal hypertension: inflammatory gastritis or congestive gastropathy? Gut. 1985;26:1226-32.

17. McCormick PA, Sankey EA, Cardin, F, Dhillon AP, Mcintyre N, Burroughs AK Congestive gastropathy and Helicobacter pylori: an endoscopic and morphometric study. Gut. 1991;32:351-4.

18. Mégraud F, Lamouliatte H. Helicobacter pylori and duodenal ulcer. Dig Dis Sci. 1992;37:769-72.

19. Nardone G, Coscione P, D'armiento FP, Del Pezzo M, Pontillo M, Mossetti G Cirrhosis negatively affects the efficiency of serologic diagnosis of Helicobacter pylori infection. Ital J Gastroenterol. 1996;28:332-6.

20. Noach LA, Bosma NB, Jansen J, Hoek FJ, van Deventer SJ, Tytigat GN. Mucosa tumor necrosis factor-alfa, interleukin-1, beta and interleukin-8 production in patients with Helicobacter pylori. Scand J Gastroenterol. 1994;29:419-24.

21. Papazian A, Braillon A, Dupas JL, Sevenet F, Capron JP. Portal hypertensive gastric mucosa: an endoscopic study. Gut. 1985;27:1199-203.

22. Pellicano R, Leone N, Berrutti M, Cutufia MA, Fiorentino M, Rizzatto M. Helicobacter pylori seroprevalence in hepatitis $\mathrm{C}$ virus positive patients with cirrhosis. J Hepatol. 2000;33:648-50. 
23. Ponzetto A, Pellicano R, Leone N, Berrutti M, Turrini F, Rizzetto M. Helicobacter pylori seroprevalence in cirrhotic patients with hepatitis B virus infection. Neth J Med. 2000;56:206-10.

24. Ponzetto A, Pellicano R, Redaelli A, Rizzetto M, Roffi L. Helicobacter pylori infection in patients with hepatitis $\mathrm{C}$ virus positive chronic liver diseases. New Microbiol. 2003;26:321-8.

25. Queiroz DMM, Rocha AMC, Rocha GA, Cinque SMS, Oliveira AG, Godoy A, Tanno H. Association between Helicobacter pylori infection and cirrhosis in patients with chronic hepatitis C virus. Dig Dis Sci. 2006;51:370-3.

26. Rabinovitz M, Yoo Y-K, Schade RR, Dindzans VJ, Van Thiel DH, Gavaler JS Prevalence of endoscopic findings in 510 consecutive individuals with cirrhosis evaluated propectively. Dig Dis Sci. 1990;35:705-10.

27. Saccheti C, Capello M, Rebecchi P, Roncucci L, Zanghieri G, Tripodi A, Ponz de Leon M. Frequency of upper gastrointestinal lesions in patients with liver cirrhosis. Dig Dis Sci. 1988;33:1218-22.

28. Sarfeh IJ, Tarnawski A, Mason GR, Mach T, Ivey RJ. Portal hypertension and gastric mucosa injury in rats. Gastroenterology. 1983;84:987-93.

29. Sarfeh IJ, Tarnawski A, Maeda R, Raymont K, Mason GR, Ivey KJ. The gastric mucosa in portal hypertension: effects of topical bile acid. Scand J Gastroenterol. 1984;9(suppl 92):189-94.

30. Sarin SK, Sreenivas DV, Lahoti D, Saraya A. Factors influencing development of portal hypertension. Gastroenterology. 1992;102:994-9.

31. Siringo S, Burrouhgs AK, Bolondi L, Muia A, Di Febo G, Miglioloi M, Cavalli G, Barbara L. Peptic ulcer and its course in cirrhosis: an endoscopic and clinical prospective study. J Hepatol. 1995;22:633-41.
32. Tsai CJ. Helicobacter pylori infection and peptic ulcer disease in cirrhosis. Dig Dis Sci. 1998;43:1219-25.

33. Tytgat GNJ. Endoscopic transmission of Helicobacter pylori. Aliment Pharmacol Ther. 1995;9(suppl 2):41-4

34. Vergara M, Calvet X, Roqué M. Helicobacter pylori is a risk factor for peptic ulcer disease in cirrhotic patients. A meta-analysis. Eur J Gastroenterol Hepatol. 2002;14:717-22.

35. Yeh JL, Peng YC, Tung CF, Chen GH, Chow WK, Chang CS, Yeh HZ, Poon SK. Role of Helicobacter pylori in cirrhotic patients with dyspepsia: a 13C-urea breath test study. Adv Ther. 2001;18:140-50.

36. Zullo A, Rinaldi V, Messi P, Folino S, Lauria V, Diana F, Winn S, Attili AF Helicobacter pylori infection in dyspeptic cirrhotic pacients. Hepatogastroenterology. 1999;46:395-400.

37. Zullo A, Hassan C, Morini S. Helicobacter pylori infection in patients with liver cirrhosis: facts and fictions. Dig Liver Dis. 2003;35:197-205.

38. Zullo A, Hassan C, Morini S. Hepatic encephalopathy and Helicobacter pylori: a critical reappraisal. J Clin Gastroenterol. 2003;37:164-8.

39. Zullo A, Romiti A, Tomao S, Hassan C, Rinaldi V, Giustini M, Morini S, Tagg F. Gastric cancer prevalence in patients with liver cirrhosis. Eur J Cancer Prev. 2004;12:179-82.

40. Zullo A, Cristofari F, Hassan C. H. Pylori should be eradicated in chronic liver disease patients with peptic ulcer. Dig Liver Dis. 2009;41:141-2.

Recebido em 12/6/2008. Aprovado em 2/3/2009. 